\title{
RESOLUTION AND EVOLUTION OF THE CORE OF THE GIANT HII REGION NGC 3603
}

\author{
K.-H. HOFMANN and G. WEIGELT \\ MPI für Radioastronomie, Bonn, Germany \\ and \\ W. SEGGEWISS \\ Observatorium Hoher List, Daun, Germany
}

\begin{abstract}
We present diffraction-limited speckle-masking observations of the central object HD 97950 in the giant H II region and star cluster NGC 3603. Twenty-eight stellar components have been detected in the $6 . " 3 \times 6 . " 3$ field from our reconstructed images. Four different filter combinations were used for the selection of distinct spectral regions comprising $\mathrm{H} \alpha$ emission, the main Wolf-Rayet and Of-type emission lines, and two continuum bands. Stellar components having WN-type characteristics have been found. A color-magnitude diagram has been constructed. Isochrone fits yield a cluster age of about 3.2 Myr, which is in accordance with the time-scale of Wolf-Rayet star evolution.
\end{abstract}

Key words: HII region NGC 3603 - stellar core HD 97950 - speckle interferometry resolution - cluster evolution

\section{Introduction}

The nature of the central object HD 97950 of the massive $\mathrm{H}$ II region NGC 3603 is still under debate. Embedded in the $\mathrm{H}$ II region is a luminous stellar cluster containing about 20 O-type and WR (WN6) stars at a distance of $7.0 \pm 0.5 \mathrm{kpc}$ and suffering $E_{\mathrm{B}-\mathrm{V}}=1.44 \pm 0.09 \mathrm{mag}$ (Moffat 1973), thus placing it in the outlying region of the Carina spiral arm $\left(l=291^{\circ} 62\right.$, $b=-0^{\circ} 52$ ). Walborn (1973) first draw attention to a number of similarities between the stellar cluster of NGC 3603 and the central cluster NGC 2070 of 30 Dor in the LMC. Especially both central objects, HD 97950 in NGC 3603 and HD 38268 (R 136a) in NGC 2070, appear as compact, superluminous WR-like objects of similar spectral type: WN6+05. Moffat, Seggewiss \& Shara (1985) made a detailed comparative study of the stellar cores of NGC 3603 and 30 Dor. They deduced that HD 97950 contains 2-3 and R136a contains 4-5 WNL stars. Spatially resolved spectra over the surface of HD 97950 indicated that both components of the central pair AB (van den Bos 1928) as well as star C might be WN6 stars. Melnick et al. (1989) presented an CCD study of NGC 3603 and found an age of $\sim 2.5 \pm 2 \mathrm{Myr}$.

The first resolution of the central core of NGC 3606 was reported by Baier et al. (1985) and Hofmann \& Weigelt (1986). In a recent HST study Moffat et al. (1994) resolved NGC 3603 and studied its star density distribution and its IMF in comparison to NGC 2070. 


\section{Observations and reduction}

NGC 3603 was observed on March 29, 1993 and January 18, 1994 with the $2.2 \mathrm{~m}$ ESO-MPG telescope at La Silla. The speckle interferograms were recorded through four filters: (1) Schott edge filter RG 695, (2) 6581/530, (3) $5453 / 253$, and (4) $4670 / 160$ (center wavelengths / bandwidth in $\AA$ ). The 6581/530 filter includes $\mathrm{H} \alpha$ (plus He II). The 5453/253 filter selects a spectral range substantially free of emission lines from Wolf-Rayet and earlytype stars and of interstellar O [III]. The 4670/160 filter picks out the He II 4686 (plus other) emission features in WR spectra.

Images with diffraction-limited resolution and high dynamic range were reconstructed by the speckle masking method (Weigelt 1977; Lohmann et al. 1983; Hofmann \& Weigelt 1986). From the speckle interferograms the average 4-dimensional bispectrum with a resolution of $\sim 24$ million elements (for all $\mathrm{u}$-vectors up to $(95,95)$ and all $\mathrm{v}$-vectors up to $(45,45)$ ) was derived. After compensation of detector noise and photon noise bias terms in the average bispectrum (Pehlemann et al. 1992), the object Fourier transform was reconstructed up to the telescope cut-off frequency.

The reconstructions obtained from the RG 695 data set and the $6581 \AA$ data set have diffraction-limited resolution, $0 . " 08$ and $0 .{ }^{\prime \prime} 075$, respectively. The reconstructions obtained from the $5453 \AA$ and $4670 \AA$ data sets have resolutions of $0 . " 073$ and $0 . " 150$, respectively. In addition to the bright components of the center of NGC 3603 discovered by earlier speckle interferometry and speckle masking observations (see above), many fainter stellar components can be seen in our new reconstructions. In the reconstructions with the highest SNR (data sets RG 695 and 6581) 28 stars were identified in the $6 . " 3 \times 6 . " 3$ field of view. The closest double component resolved in the reconstructions from data sets (1), (2) and (3) has a separation of $0 .{ }^{\prime \prime} 074$. The intensity ratio of the brightest to the faintest components is approximately $100: 1$. Fig. 1 shows the diffraction-limited speckle reconstruction obtained from the $6581 \AA$ data.

\section{Photometry}

The four filters used in our investigation define a system of four magnitudes. Though the bandwidth of the 5453 filter is considerably narrower than the $V$ passband of the Johnson $U B V$ system, the magnitudes in this band are a fairly good representation of Johnson $V$, allowing an absolute calibration of the relative (instrumental) $V$ magnitudes in the center of NGC 3603 (HD 97950): van den Bergh (1978) derived $V=10.02 \mathrm{mag}$ for the inner $5^{\prime \prime}$ of HD 97950. We take this value as the integrated $V$ magnitude for the 5453 band of all detected 20 stars in a circle of $5^{\prime \prime}$ diameter around the central star and can now calculate individual magnitudes. 


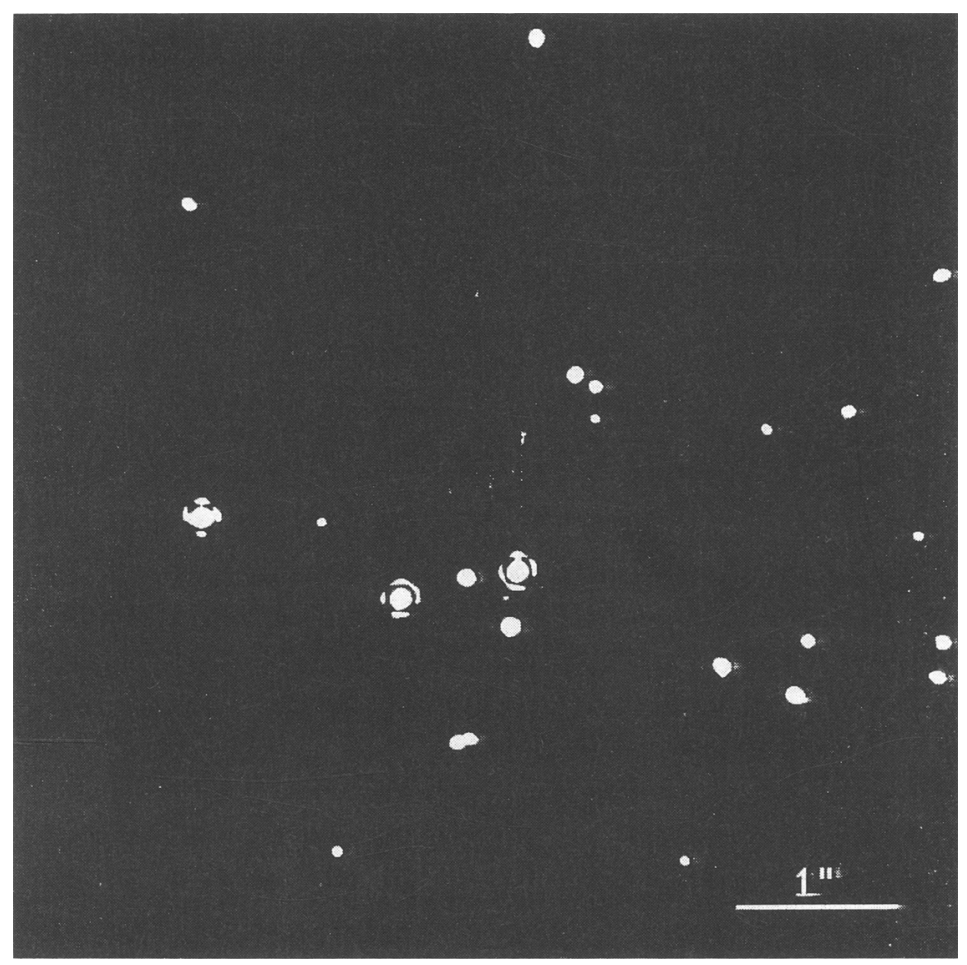

Fig. 1. Diffraction-limited image of NGC 3603 reconstructed from the RG 695 data set (2269 frames, 8150 photoevents per frame).

\section{The evolutionary state}

The 4670 filter measurements allows to estimate which components of HD 97950 have Wolf-Rayet characteristics. Unfortunately only the brightest stars could be detected in this spectral range and the errors of these data are the largest in our four data sets. With this reservations in mind we derive from the 4670/5453 two-color diagram that stars \#2 and \#5 have strong WN characteristics and stars \#1 and \#3 have mild WN characteristics. This means that the latter stars could be of type Of, i.e., in the transition stage between the main sequence and WN-type stars.

With the speckle-detected components of HD 97950 we can construct the CMD of NGC 3603 (Fig. 2) which is now complete at its top. [The $(B-V)$ values of the speckle-detected stars are, for obvious reasons, assumed to be $1.06 \mathrm{mag}$, i.e., equal to van den Bergh's (1978) integrated $(B-V)$ value for the inner $5^{\prime \prime}$ of NGC 3603.] For stars in the surrounding of the centre we 


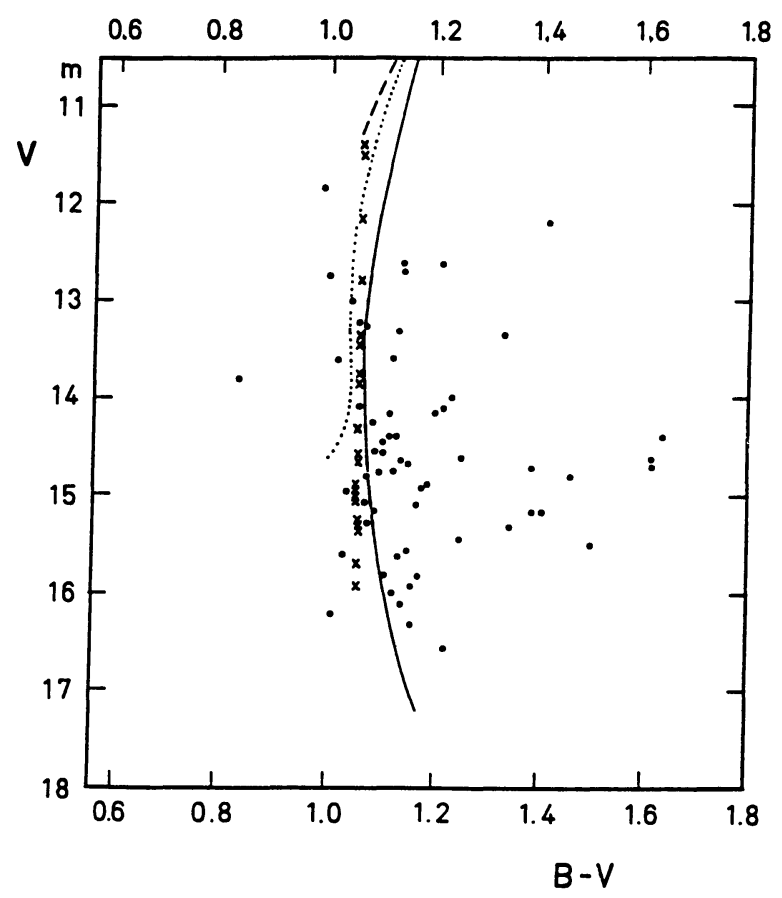

Fig. 2. CMD of the cluster NGC 3603. The crosses are speckle-detected star in the cluster centre, the dots are stars surrounding the centre (Melnick et al. 1989). The lines represent the isochrone with age 3.2 Myr, $Y=0.30$, and $Z=0.02$ (Meynet et al. 1993)

take the data from Melnick et al. (1989). The brightest stars in the CMD are the central components of HD 97950, both bearing WN characteristics. The brightest star of the surrounding is star Sher 67 (Sher 1965) with spectral type 05.5III(f) (Moffat 1983); its spectrum indicates that the star is in the transitional phase between main-sequence and WN state.

We have made an isochrone fit to the CMD of NGC 3603 using the isochrones published by Meynet et al. (1993). The isochrone with near-solar composition $(Y=0.30$ and $Z=0.02$ ) and $\log$ age $=6.5$ (i.e., $3.2 \mathrm{Myr}$ ) gives the best fit to the diagram. We derive a reddening of $E_{\mathrm{B}-\mathrm{V}}=1.38$ and a distance modulus of $V-M_{V}=14.18$ or a distance $d=6.9 \mathrm{kpc}$. The values of reddening and distance modulus are slightly smaller than earlier determinations.

The age of about $3 \mathrm{Myr}$ is supported by the presence of WN-type stars: The stellar models of Schaller et al. (1992) demand an lower age limit of 2.8 Myr for the first appearance of a WNL star with progenitor mass $85 \mathcal{M}_{\odot}$ and an age of $3.5 \mathrm{Myr}$ for $60 \mathcal{M}_{\odot}$. Thus, an age of $3 \mathrm{Myr}$ agrees with the 
model WR star evolution, if we do not evoke extraordinarily high masses $\left(>90 \mathcal{M}_{\odot}\right)$ for the WN progenitors in the centre of NGC 3603. On the other hand, no red supergiants can be found in the clusters which poses an upper age limit of about $7 \mathrm{Myr}$.

The 3.2 Myr isochrone is fairly complicated in its evolved high mass part. It has a loop in its WNL phase (dashed line in the upper part of Fig. 2) which is followed by a transition from WNL over WNE to the WC phase (descending dotted line in Fig. 2). The central stars lie just at the faint end of the WNL loop.

\section{References}

Baier, G., Ladebeck, R., Weigelt, G. 1985, $A \mathscr{E} A 151,61$

Hofmann, K.-H., Weigelt, G. 1986, $A \& A$ 167, L15

Lohmann, A.W., Weigelt, G., Wirnitzer, B. 1983, Appl. Opt. 22, 4028

Melnick, J., Tapia, M., Terlevich, R. 1989, $A \& A$ 213, 89

Meynet, G., Mermilliod, J.-C., Maeder, A. 1993, A\&A Suppl. 98, 477

Moffat, A.F.J. 1983, $A \mathscr{G} A$ 124, 273

Moffat, A.F.J., Drissen, L., Shara, M.M. 1994, $A p J$ in press

Moffat, A.F.J., Seggewiss, W., Shara, M.M. 1985, ApJ 295, 109

Pehlemann, E., Hofmann, K.-H., Weigelt, G. 1992, $A \& A$ 256, 701

Schaller, G., Schaerer, D., Meynet, G., Maeder, A. 1992, A\&A Suppl. 96, 269

Sher, D. 1965, MNRAS 129, 237

van den Bergh, S. 1978, $A \& A$ 63, 275

van den Bos, W.H. 1928, Bull. Astron. Inst. Neth. 4, 261

Walborn, N.R. 1973, ApJ 182, L21

Weigelt, G.P. 1977, Opt. Commun. 2155

\section{DISCUSSION:}

Moffat: As you mentioned, we (Moffat, Drissen, Shara) have a paper in press (ApJ, 1994) giving HST-PC observations from 1991/92 of NGC 3603. These results concur with your speckle data. The "new" HST will, of course, do much better (e.g. reaching much fainter stars in a $60^{\prime \prime} \times 60^{\prime \prime}$ region at $\checkmark 0^{\prime \prime} .06$ resolution). Also we have HST time to obtain spectra of individual stars right in the core of $\mathrm{HD} 97950$.

Another important similarity between R136 in 30 Dor and NGC 3603 is their stellar density profile, reaching very high densities in their cores. This makes NGC 3603 a $7 x$ closer clone of R136, with NGC 3603 very much easier to study. However, an important difference between NGC 3603 and R136 is the large stellar halo (cluster NGC 2070) around R136. NGC 3603 is thus a naked core by comparison, for reasons that must be related to different starburst formation mechanisms in the LMC compared to the Galaxy.

Seggewiss: Let me point out that the resolution also of the "new" HST is limited by diffraction to about $0 " .07$ ! This limit might be pushed down a little bit by modern image evaluation techniques.

Gies: What is the spatial density (number of stars per cubic parsec) in the central 6 arcsec of the cluster?

Moffat: ...about like what one finds in the corner of the densest globular clusters, if one extrapolates the IMF to faint star using, say, Salpeter. 